\title{
ARTICLE
}

\section{Increased risk of diseases of the basal ganglia and cerebellum in patients with a history of attention-deficit/hyperactivity disorder}

\author{
Karen Curtin ${ }^{1}$, Annette E. Fleckenstein ${ }^{2}$, Brooks R. Keeshin ${ }^{3,4}$, Deborah A. Yurgelun-Todd ${ }^{5}$, Perry F. Renshaw ${ }^{5}$, Ken R. Smith ${ }^{6}$ and \\ Glen R. Hanson ${ }^{2}$
}

\begin{abstract}
Attention-deficit/hyperactivity disorder (ADHD) is marked by an ongoing pattern of inattention and/or hyperactivity and involves dysregulated dopaminergic pathways. Dopaminergic agents (i.e., amphetamine and methylphenidate) are thus prescribed to treat ADHD. As little is known regarding long-term consequences of either ADHD or its treatment, the objective of this study was to determine if either alters the risk of diseases of the basal ganglia and cerebellum, including Parkinson's disease. Statewide medical records from 1996 to 2016 were retrieved from the Utah Population Database to conduct a retrospective cohort study. Participants included ADHD patients (International Classification of Diseases, 9th revision (ICD-9) diagnosis codes 314.0-314.2, 314.8, 314.9) and 5:1 random sex-matched and age-matched subjects with no ADHD diagnosis history. Both patients and non-ADHD subjects met the following eligibility criteria: (1) no prior diagnosis of Parkinson's disease, secondary parkinsonism, basal ganglia disease, or essential tremor (ICD-9 codes 332.0, 332.1, 333.0, 333.1), (2) born in 1950 or later and age $\geq 20$ years at last follow-up, and (3) no history of substance abuse (illicit drugs or alcohol). Outcomes were measured as time to diagnosis of diseases of the basal ganglia and cerebellum, death, or study-end. A Cox model incorporating a competing risk of death was used to provide hazard ratio estimates. Patients with ADHD $(N=31,769)$ had a 2.4-fold increased risk of basal ganglia and cerebellum diseases (95\% confidence interval (Cl): $2.0-3.0 ; P<0.0001)$ compared with 158,790 non-ADHD persons, after controlling for sex and age and adjusting for tobacco use and psychotic conditions. In 4960 ADHD patients prescribed psychostimulants, risk of basal ganglia and cerebellum diseases between ages 21 and 49 years was especially pronounced, at 8.6-fold (95\% Cl: 4.8-15.6; $P<0001)$. The association of ADHD patients prescribed psychostimulants with higher risk of diseases of the basal ganglia and cerebellum may reflect a more severe ADHD phenotype rather than a direct association between prescribed stimulant use and basal ganglia or cerebellum disorders. Future studies to assess and stratify patient risk so as to inform treatment are warranted.
\end{abstract}

Neuropsychopharmacology (2018) 43:2548-2555; https://doi.org/10.1038/s41386-018-0207-5

\section{INTRODUCTION}

Attention-deficit/hyperactivity disorder (ADHD) is a brain disorder marked by an ongoing pattern of hyperactivity-impulsivity and/or inattention that interferes with function or development. Symptoms generally appear in childhood, and often persist into adulthood [1-3]. According to the Centers for Disease Control and Prevention (CDC) approximately $11 \%$ of children $4-17$ years of age (6.4 million) have been diagnosed with the disorder. A recent CDC report of children in the United States covered by employersponsored insurance or Medicaid indicates that $75 \%$ of young children under care for ADHD received medications, usually psychostimulants such as amphetamine-based (AMPH) or methylphenidate-based products [4].

Preclinical studies have demonstrated that high-dose exposure to AMPH or its analog, methamphetamine (METH), causes persistent basal ganglia dopaminergic deficits [5]. In clinical studies, basal ganglia dopaminergic deficits have been demonstrated in individuals with a history of METH abuse [6-9], and are of particular interest given epidemiological reports that abusers of AMPH/METH are more likely to develop Parkinson's disease [10-12]. However, little is known about the effects of longterm, therapeutic use of psychostimulants such as that associated with treatment of ADHD. Using the Utah Population Database (UPDB), we similarly reported that individuals with a history of AMPH/METH abuse were more likely to develop basal ganglia and cerebellum (BG\&C) disorders including: Parkinson's disease, secondary parkinsonism, other diseases of the basal ganglia, and/or essential tremor [13]. Based on these findings and the fact that $\mathrm{AMPH}$-like drugs are linked to both prescribed first-line treatment for the management of $\operatorname{ADHD}[4,14,15]$ and nonprescribed self-administration of these agents $[16,17]$, the present study investigates if a diagnosis of ADHD may be linked to increased incidence of BG\&C diseases, including Parkinson's disease. Of particular interest was risk of $B G \& C$ diseases occurring

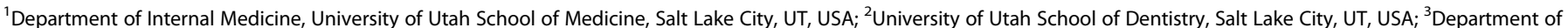

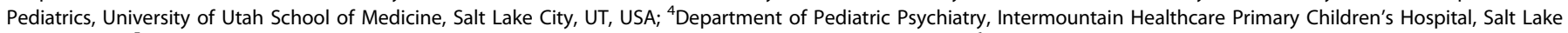

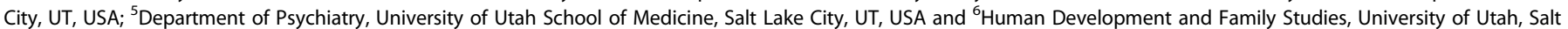
Lake City, UT, USA

Correspondence: Glen R. Hanson (glen.hanson@hsc.utah.edu)

Received: 16 March 2018 Revised: 10 August 2018 Accepted: 28 August 2018

Published online: 12 September 2018 
Table 1. ICD-9 diagnosis codes

\begin{tabular}{|c|c|}
\hline ICD-9 codes & Description \\
\hline \multicolumn{2}{|l|}{ I. Population exclusions } \\
\hline 042, V08 & HIV disease or infection \\
\hline $303,305.0$ & $\begin{array}{l}\text { Alcohol dependence syndrome, } \\
\text { nondependent alcohol abuse }\end{array}$ \\
\hline $304.0,305.5$ & Opioid dependence or abuse \\
\hline $304.1,305.4$ & $\begin{array}{l}\text { Barbiturates and sedative dependence } \\
\text { or abuse }\end{array}$ \\
\hline $304.2,305.6$ & Cocaine dependence or abuse \\
\hline $304.3,305.2$ & Cannabis dependence or abuse \\
\hline $304.4,305.7$ & $\begin{array}{l}\text { Amphetamine and psychostimulant } \\
\text { dependence or abuse }\end{array}$ \\
\hline $304.5,305.3$ & Hallucinogen dependence or abuse \\
\hline $304.7,304.8$ & $\begin{array}{l}\text { Combinations of opioid, non-opioids } \\
\text { with other drug dependence }\end{array}$ \\
\hline $304.6,304.9,305.9$ & $\begin{array}{l}\text { Other specified or unspecified drug } \\
\text { dependence or drug abuse }\end{array}$ \\
\hline 965.0 & $\begin{array}{l}\text { Poisoning by opiates and related } \\
\text { narcotics }\end{array}$ \\
\hline 968.5 & $\begin{array}{l}\text { Surface and infiltration anesthetics } \\
\text { including cocaine }\end{array}$ \\
\hline 969.6 & Poisoning by cannabis \\
\hline 969.7 & Poisoning by psychostimulants \\
\hline 980.0 & Toxic effect of ethyl alcohol \\
\hline E850.0-E850.2 & $\begin{array}{l}\text { Accidental poisoning by heroin, } \\
\text { methadone, or opiates }\end{array}$ \\
\hline E854.1 & Accidental poisoning by cannabis \\
\hline E854.2 & $\begin{array}{l}\text { Accidental poisoning by } \\
\text { psychostimulants }\end{array}$ \\
\hline E855.2 & Accidental poisoning by cocaine \\
\hline E860.0, E860.1 & $\begin{array}{l}\text { Accidental poisoning by alcoholic } \\
\text { beverages or ethyl alcohol }\end{array}$ \\
\hline
\end{tabular}

II. ADHD patients

314.00, $314.01 \quad$ Attention-deficit disorder without or with mention of hyperactivity

314.1 Hyperkinesis with developmental delay

314.2 Hyperkinetic conduct disorder

314.8, $314.9 \quad$ Other or unspecified manifestations of hyperkinetic syndrome

III. Basal ganglia and cerebellum diseases

$\begin{array}{ll}332.0 & \begin{array}{l}\text { Parkinson's disease, paralysis agitans } \\ \text { Parkinson's disease, secondary } \\ \text { parkinsonism }\end{array} \\ 333.0 & \begin{array}{l}\text { Other degenerative diseases of the } \\ \text { basal ganglia } \\ \text { Essential and other specified forms of } \\ \text { tremor }\end{array}\end{array}$

IV. Psychotic conditions

$\begin{array}{ll}290 & \text { Dementias } \\ 291 & \text { Alcohol-induced mental disorders } \\ 292 & \text { Drug-induced mental disorders } \\ 293,294 & \text { Disorders due to conditions classified } \\ & \text { elsewhere } \\ 295 & \text { Schizophrenic disorders } \\ 296 & \text { Episodic mood disorders } \\ 297 & \text { Delusional disorders } \\ 298 & \text { Other nonorganic psychoses } \\ 299 & \text { Pervasive developmental disorders }\end{array}$

\begin{tabular}{|ll|}
\hline Table 1 continued & \\
\hline ICD-9 codes & Description \\
\hline V. Tobacco use & \\
305.1 & Current tobacco use \\
989.84 & Toxic effect of tobacco \\
V15.82 & History of tobacco use \\
Generic name & Trade name \\
VI. ADHD medications & \\
Methylphenidate & Concerta, Daytrana, Metadate (CD/ER), \\
& Methylin (ER), Ritalin (LA/SR) \\
Dexmethylphenidate & Focalin (XR) \\
Mixed amphetamine salts & Adderall (XR) \\
\hline ICD-9 International classification of disease, 9th revision, HIV human \\
immunodeficiency virus & \\
\hline
\end{tabular}

in those aged 21-49 years, an age group previously identified as defining young onset Parkinson's disease [18]. We also explore if factors potentially related to BG\&C diseases: tobacco use, psychotic conditions, or demographic factors (race/ethnicity and sex) modify our observations.

\section{METHODS}

Utah Population Database

Located at the University of Utah, the UPDB contains computerized records of over 11 million individuals who currently reside in or have lived in Utah as described; see Supplement, Appendix A for details. The UPDB includes vital records, statewide inpatient discharge data, and links to clinical records of patients in the University of Utah and Intermountain Healthcare systems. With a combined 26 hospitals and 220 clinics, these two systems account for approximately $85 \%$ of patient encounters in Utah annually. Electronic medical records (EMRs) from 1996 include International Classification of Diseases, 9th revision (ICD-9) codes and pharmacy orders. Approvals and waivers of consent to conduct this recordbased study were granted from the University of Utah Institutional Review Board (IRB 00052716), Intermountain Healthcare Research Institutional Review Board (IRB 1024204), and the governing body of UPDB data, the Resource for Genetic Epidemiologic Research (RGE 00000568).

\section{Source population}

In this retrospective cohort study, the source population is comprised of all Utah individuals born between 1950 and 1992. Given a clinical definition of ADHD was not officially published by the American Psychiatric Association until 1968 in the second edition of the Diagnostic and Statistical Manual of Mental Disorders (DSM II) and ADHD often manifests during childhood or adolescence [1], it is likely that ADHD would have been substantially underdiagnosed in those born before 1950. Therefore, our source population was defined to better match the time period in which ADHD became a clinically recognized disorder. Study inclusion criteria were: (1) at least 20 years of age on 31 December 2011 or date of last follow-up, if earlier and (2) resident of Utah on or after 1 January 1996 (baseline) as determined by vital records and other demographic data in the UPDB. As human immunodeficiency virus (HIV) can result in Parkinson-like symptoms [19], we excluded 1550 individuals with an HIV diagnosis who otherwise met the eligibility. To avoid potential confounding from substance use disorders, we further excluded 105,493 individuals with any history of abuse of AMPH/METH, other illicit drugs, or alcohol abuse based on an ICD-9 diagnosis (Table 1, section I) in the EMR. These confounds were selected because a history of AMPH/METH abuse has been associated with Parkinson's disease [11, 13, 20], and among illicit 
drug abusers, use of multiple substances is commonplace [21] and may not be reflected in the EMR. Although inconsistent findings in a recent review suggest a complex relationship between alcohol use and Parkinson's disease [22], moderate/heavy liquor consumption (vs. beer or wine) was reported to prospectively increase Parkinson's disease risk [23, 24], and thus we also excluded individuals with alcohol abuse disorders. The resulting source population included 1.92 million individuals.

ADHD patient cohort

From the source population, 32,740 individuals with an ICD-9 diagnosis of attention-deficit (with or without hyperactivity) or other hyperactivity disorders including: hyperkinesis with developmental delay, hyperkinetic conduct disorder, and other or unspecified manifestations of hyperkinetic syndrome (Table 1, section II) appearing in one or more EMR from 1996 to 2011 were assigned to the ADHD patient cohort. The earliest diagnosis date in the medical record was designated as the index diagnosis. Patients were excluded for the following: (1) missing follow-up in Utah after an index diagnosis $(n=884)$, (2) a diagnosis of any $B G \& C$ disorder that occurred prior to an index ADHD diagnosis $(n=63)$, or (3) any BG\&C disease diagnosis before age 21 years $(n=24)$ such as juvenile Parkinson's which is a rare condition attributed to hereditary mutations or metabolic conditions, and is atypical of idiopathic Parkinson's disease $[25,26]$. The resulting ADHD cohort included 31,769 patients. From a text-based search of formulary and trade names (Table 1, section $\mathrm{VI}$ ) in pharmacy orders records of Intermountain Healthcare and University of Utah Healthcare facilities, a subset of 4960 ADHD patients within the cohort of 31,769 patients were determined to have been prescribed stimulant treatment: mixed-AMPH salts $(n=2716)$, methylphenidate $(n=1941)$, or both agents $(n=303)$.

\section{Non-ADHD cohort}

A cohort of 158,790 individuals with no diagnosis history of ADHD and no diagnosis of $B G \& C$ diseases prior to baseline were randomly selected from the source population and individually matched to ADHD patients based on sex and birth year at a target ratio of 5:1. Non-ADHD subjects were required to have follow-up in Utah from baseline until at least the index diagnosis date of their respective matched ADHD patient.

Outcome of diseases of the basal ganglia and cerebellum We measured time from baseline to an index diagnosis on or before 31 December 2016 of any adult-onset diseases of the basal ganglia and cerebellum including Parkinson's disease, secondary parkinsonism, other degenerative diseases of the basal ganglia, and essential tremor based on ICD-9 codes (or ICD-10 equivalent beginning October 2015; see Table 1, section III). Hereafter, diseases of the basal ganglia and cerebellum are referred to as $B G \& C$ diseases. Right censoring occurs when a subject dies, is lost to follow-up, or the study ends before an outcome is observed. Study subjects with no diagnosis of BG\&C diseases who died on or before 31 December 2016 were treated as having a competing cause of death and right censored at their death date. Subjects alive on 31 December 2016 (or at last follow-up, if earlier) who did not develop BG\&C diseases were right censored.

Statistical methods

A Cox proportional hazards model was used to calculate a hazard ratio (HR) estimate of the risk of an incident diagnosis of BG\&C diseases in ADHD patients compared with non-ADHD subjects. To account for matching of non-ADHD to ADHD individuals, separate hazard functions (i.e., stratified Cox regressions) were specified for each matched group consisting of an ADHD patient and five associated non-ADHD subjects matched on sex and birth year. $A$ competing risk of death was incorporated [27] as ADHD is associated with excess mortality from unnatural causes [28] and
BG\&C diseases, such as Parkinson's disease, are typically lateronset disorders.

\section{Model covariates}

The model included covariate adjustment for self-reported race/ ethnicity (white and non-Hispanic, white and Hispanic, or nonwhite) and for potentially confounding factors that could directly influence the expression of BG\&C diseases. These were: (1) psychotic conditions as a proxy for antipsychotics and that conditions such as schizophrenia are believed to be caused by excessive dopaminergic activity [29] and (2) tobacco use via smoking, because of its reported association with decreased risk of Parkinson's disease [30,31]; however, there is some controversy as to the appropriate interpretation of some of these tobaccorelated epidemiological data [32]. To address the first confound of psychotic comorbidities and potential use of neuroleptics which can cause extrapyramidal-related side effects [33], an indicator of presence or absence of an ICD-9 diagnosis of psychotic conditions (Table 1, section IV) was included as a covariate. To address the second confound of tobacco use, an indicator of presence or absence of an ICD-9 diagnosis of tobacco use (Table 1, section V) was also incorporated. In addition to the independent effects of covariates, evidence of a statistical interaction (multiplicative scale) between each pair of covariates and between each covariate and ADHD status was examined.

\section{Study analyses}

We report the adjusted HR (aHR) and 95\% confidence intervals (Cls) for risk of BG\&C diseases in ADHD patients compared with non-ADHD subjects controlling for sex, age, race/ethnicity, psychotic conditions, tobacco use, and an interaction of psychotic conditions and ADHD status $\left(P_{\text {int }}=0.001\right)$. We separately assessed risk in those ADHD patients known to be prescribed stimulant medications and those patients with no known treatment compared to their individually matched non-ADHD mates. In a parallel analysis, we examined the risk of Parkinson's disease (ICD9332.0 only) in patents vs. non-ADHD to examine BG\&C diseases exclusive of a diagnosis based on ICD-9 codes for secondary parkinsonism, other degenerative diseases of the basal ganglia, or essential tremor. All $P$ values presented were adjusted for multiple comparisons using a Bonferroni-corrected significance of $P \leq 0.005$ based on 10 independent tests. All statistical analyses were performed using SAS ${ }^{\circledast}$ version 9.4 statistical software (SAS Institute Inc., Cary, NC, USA).

\section{RESULTS}

Study characteristics

The characteristics of 158,790 non-ADHD subjects and 31,769 ADHD patients are shown in Table 2. Overall, ADHD was diagnosed more often in men than in women (57.3 vs. $42.7 \%$ ). The median index age at which a diagnosis of ADHD first appeared in the EMR in our study was 26 years. In many patients, it is likely that ADHD onset occurred at a much younger age than the median index age. A similar majority of non-ADHD and ADHD were white and non-Hispanic ( 88.8 and $89.9 \%$, respectively). The Utah population is comprised primarily of Caucasians of northern European descent; in the 2010 census, $86 \%$ of those who selected only one race ( $97 \%$ of the Utah population) reported as Caucasian. ADHD patients were more likely to have died during the study than non-ADHD subjects, consistent with increased mortality reported in ADHD patients [28]. Median follow-up was 21 years over the 1996-2016 study period. Consistent with Utah's tobacco use profile in non-ADHD subjects, 150,037 (94.5\%) had no personal tobacco use history, while in ADHD patients, 29,032 $(91.4 \%)$ had no history of tobacco use. Concerning psychotic comorbidities, ADHD patients were more likely than non-ADHD subjects to have had a psychotic condition diagnosed (12.6 and 
Table 2. Characteristics of non-ADHD subjects and ADHD patients

\begin{tabular}{|c|c|c|c|c|c|}
\hline \multirow[t]{3}{*}{ Discrete } & \multicolumn{5}{|c|}{ Characteristics } \\
\hline & \multicolumn{2}{|c|}{$\begin{array}{l}\text { Matched non- } \\
\text { ADHD subjects }\end{array}$} & \multicolumn{2}{|l|}{$\begin{array}{l}\text { ADHD } \\
\text { patients }\end{array}$} & \multirow[t]{2}{*}{$P$ value } \\
\hline & $N$ & Col\% & $N$ & Col\% & \\
\hline All subjects & 158,790 & 100.0 & 31,769 & 100.0 & - \\
\hline \multicolumn{6}{|l|}{ Sex } \\
\hline Females & 67,802 & 42.7 & 13,564 & 42.7 & \\
\hline Males & 90,988 & 57.3 & 18,205 & 57.3 & - \\
\hline \multicolumn{6}{|l|}{ ADHD treatment status } \\
\hline+ stimulants & - & - & 4960 & 15.6 & \\
\hline None or unknown & - & - & 26,809 & 84.4 & - \\
\hline \multicolumn{6}{|l|}{ Vital status } \\
\hline Died during follow-up & 1248 & 0.8 & 716 & 2.3 & \\
\hline Alive at last follow-up & 157,542 & 99.2 & 31,053 & 97.7 & $<0.001$ \\
\hline \multicolumn{6}{|l|}{ Psychiatric conditions } \\
\hline Diagnosis history & 3336 & 2.1 & 4005 & 12.6 & \\
\hline No diagnosis history & 155,454 & 97.9 & 27,764 & 87.4 & $<0.001$ \\
\hline \multicolumn{6}{|l|}{ Tobacco use history } \\
\hline Diagnosis history & 8753 & 5.5 & 2737 & 8.6 & \\
\hline No diagnosis history & 150,037 & 94.5 & 29,032 & 91.4 & $<0.001$ \\
\hline \multicolumn{6}{|l|}{ Race and ethnicity } \\
\hline White and non-Hispanic 1 & 141,071 & 88.8 & 28,550 & 89.9 & \\
\hline Non-white or Hispanic & 17,719 & 11.2 & 3219 & 10.1 & $<0.001$ \\
\hline Continuous & $\begin{array}{l}\text { Mean } \\
( \pm \text { s.d. })\end{array}$ & & Median $(r)$ & $\begin{array}{l}\text { Mear } \\
( \pm \text { s.d }\end{array}$ & \\
\hline Years of follow-up $21(1-21)$ & $20.9( \pm$ & $.5)$ & $21(1-21)$ & 20.8 & $( \pm 1.2)-$ \\
\hline $\begin{array}{l}\text { ADHD index age - } \\
\text { (years) }\end{array}$ & - & & $26(3-61)$ & $\begin{array}{l}28.2 \\
( \pm 12\end{array}$ & \\
\hline
\end{tabular}

${ }^{a} X^{2}$ test, discrete data; paired $t$ test, continuous data; ns, not significant at the 0.05 level

$2.1 \%$, respectively). Medications prescribed to treat ADHD patients were available for a subset of 4960 ADHD patients $(15.6 \%$ of all ADHD) who were individually matched to 24,792 non-ADHD subjects (Table 2). In patients known to have stimulant treatment, mixed-AMPH salts were prescribed for 2716 patients $(55 \%)$, while methylphenidate was prescribed for 1941 patients (39\%). In $6 \%$ of ADHD patients, both AMPH salts and methylphenidate were known to be prescribed.

The number of incident outcomes of BG\&C diseases observed in ADHD patients and their associated unexposed subjects between 1996 and 2016 are shown in Table 3. In non-ADHD subjects and ADHD patients, the rates of incident BG\&C diseases were $0.19 \%$ and $0.52 \%$, respectively. Given study individuals were born between 1950 and 1992, onset of BG\&C diseases occurred between ages 21 and 66 years. Median age at an index diagnosis of BG\&C diseases was slightly younger in ADHD patients (43 years) than in non-ADHD subjects (45 years). In ADHD patients, an index diagnosis of $B G \& C$ diseases occurred on average 6.6 years after an index diagnosis of ADHD. In matched non-ADHD subjects, a diagnosis of BG\&C diseases occurred on average 5.4 years after their corresponding ADHD patient was diagnosed.

Risk of diseases of the basal ganglia and cerebellum Shown in Fig. 1 are aHRs and 95\% Cls for risk of incident BG\&C diseases in the ADHD patient cohort for: (1) all ADHD patients, (2) those patients known to be prescribed stimulants (AMPH-based and/or methylphenidate-based agents) for ADHD treatment, (3) those patients known to be prescribed only methylphenidate for ADHD treatment, and (4) patients with no known stimulant treatment, compared with their respective, matched non-ADHD subjects. Overall, ADHD was associated with a 2.4-fold increased risk of $\mathrm{BG} \& \mathrm{C}$ diseases (aHR 2.4, 95\% Cl: 2.0-3.0; $P<0.0001$ ). In ADHD patients known to be prescribed stimulants for treatment of ADHD compared with their respective non-ADHD-matched subjects (Fig. 1), risk of BG\&C diseases was significantly greater (aHR $=6.0,95 \% \mathrm{Cl}: 3.9-9.1 ; P<0.0001)$ than for ADHD patients without known stimulant treatment $(\mathrm{aHR}=1.8,95 \% \mathrm{Cl}$ : $1.4-2.3$; $P<0.0001$ ). Non-overlapping confidence intervals indicate patients known to be prescribed stimulants and those not known to receive stimulants differed in BG\&C diseases risk (Fig. 1). Because of the apparent enhancement of risk for developing $B G \& C$ diseases when ADHD patients were known to be prescribed stimulants, we determined if methylphenidate therapy, in particular, contributed to this observation as it has been reported in animal models that methylphenidate appears to protect against drug-induced dopaminergic deficits [34]. As shown in Fig. 1, ADHD patients who were only prescribed methylphenidate for treatment had an eightfold increased risk of BG\&C diseases compared to their corresponding non-ADHD subjects $(\mathrm{aHR}=8.0,95 \% \mathrm{Cl}$ : $4.2-15.1 ; P<0.0001)$. This risk was significantly greater than the 2.4-fold risk that was observed in ADHD patients generally, or the 1.8-fold risk observed in ADHD patients without known stimulant treatment. Risk of BG\&C diseases with onset at ages 21-49 in ADHD patients without known stimulant treatment was similar to that of all ADHD patients (Fig. 2). However, the risk of BG\&C diseases in ADHD patients prescribed stimulants appeared to be particularly pronounced compared to non-ADHD in this younger population (aHR $=8.6,95 \% \mathrm{Cl}: 4.8-15.6 ; P<0.0001)$.

Risk of Parkinson's disease

In a corollary analysis, we assessed risk of Parkinson's disease (ICD9 332.0) in ADHD patients compared to non-ADHD persons (Fig. 3). Of subjects with a BG\&C diseases outcome, 96 of nonADHD (32.3\%) and 56 of ADHD patients (33.7\%) had an ICD-9 diagnosis of Parkinson's disease. Although power to detect an association was reduced given fewer outcomes, a 2.6-fold increased risk of Parkinson's disease was observed for ADHD patients compared with non-ADHD (aHR 2.6, 95\% Cl: 1.8-3.7; $P<$ 0.0001 ) consistent with risk of BG\&C diseases (including Parkinson's disease; see Fig. 1). In ADHD patients known to have been prescribed medications, risk of Parkinson's disease was $\sim 4$-fold that of non-ADHD subjects (aHR 3.9, 95\% Cl: 1.9-8.3; $P=0.003$ ). However, the confidence interval overlapped with that of patients without known stimulant treatment (aHR 2.3, 95\% Cl: 1.5-3.5; $P=$ 0.0007). Crude HRs from a parallel set of models accounting for matching on sex and age, but unadjusted for covariates of psychotic conditions and tobacco use were consistent with the adjusted model results across analyses; see Figures S1-S3 in an online supplement).

\section{DISCUSSION}

In our population-based investigation, we observed an increased risk for expression of BG\&C diseases in persons with a history of ADHD compared to individuals with no ADHD history. Specifically, individuals with an ADHD diagnosis had a more than twofold increased risk of a subsequent diagnosis of BG\&C diseases (including Parkinson's disease, secondary parkinsonism, other degenerative diseases of the basal ganglia, and essential tremor) over 20 years of follow-up. Furthermore, when restricting the outcome of interest to Parkinson's disease (ICD-9 332.0), our finding of increased risk in ADHD patients was of comparable magnitude. These results are consistent with the hypothesis that an increase in expression of BG\&C diseases, including Parkinson's 
Table 3. Diseases of the basal ganglia and cerebellum outcomes in non-ADHD subjects and ADHD patients

\begin{tabular}{|c|c|c|c|c|c|c|c|c|c|c|c|c|c|c|}
\hline & \multicolumn{7}{|c|}{ Matched non-ADHD subjects } & \multicolumn{7}{|c|}{ ADHD patients } \\
\hline & \multirow{2}{*}{$\begin{array}{l}\text { Total } \\
N\end{array}$} & \multicolumn{2}{|c|}{$B G \& C$} & \multicolumn{2}{|c|}{$B G \& C<50$ y } & \multicolumn{2}{|l|}{ PD } & \multirow{2}{*}{$\begin{array}{l}\text { Total } \\
N\end{array}$} & \multicolumn{2}{|c|}{$B G \& C$} & \multicolumn{2}{|c|}{$B G \& C<50 y$} & \multicolumn{2}{|l|}{ PD } \\
\hline & & $N$ & Row\% & $N$ & Row\% & $N$ & Row\% & & $N$ & Row\% & $N$ & Row\% & $N$ & Row\% \\
\hline All subjects & 158,790 & 297 & 0.19 & 181 & 0.11 & 96 & 0.06 & 31,769 & 166 & 0.52 & 111 & 0.35 & 56 & 0.18 \\
\hline \multicolumn{15}{|l|}{ Sex } \\
\hline Females & 67,802 & 154 & 0.23 & 91 & 0.13 & 42 & 0.06 & 13,564 & 84 & 0.62 & 56 & 0.41 & 31 & 0.23 \\
\hline +stimulants & 24,792 & 56 & 0.23 & 26 & 0.10 & 19 & 0.08 & 4960 & 62 & 1.25 & 42 & 0.85 & 19 & 0.38 \\
\hline None/unknown & 133,998 & 241 & 0.18 & 155 & 0.12 & 77 & 0.06 & 26,809 & 104 & 0.39 & 69 & 0.26 & 38 & 0.14 \\
\hline & \multicolumn{5}{|c|}{ Median $(r)$} & \multicolumn{2}{|c|}{ Mean ( \pm s.d.) } & \multicolumn{5}{|c|}{ Median $(r)$} & \multicolumn{2}{|c|}{ Mean ( \pm s.d.) } \\
\hline
\end{tabular}

$B G \& C$ diseases of the basal ganglia and cerebellum, $y$ years, $<50 y$ BG\&C onset at ages 21-49 years, PD Parkinson's disease (ICD-9 332.0)

${ }^{\text {a }}$ For the non-ADHD cohort, subjects were matched to their respective ADHD patients

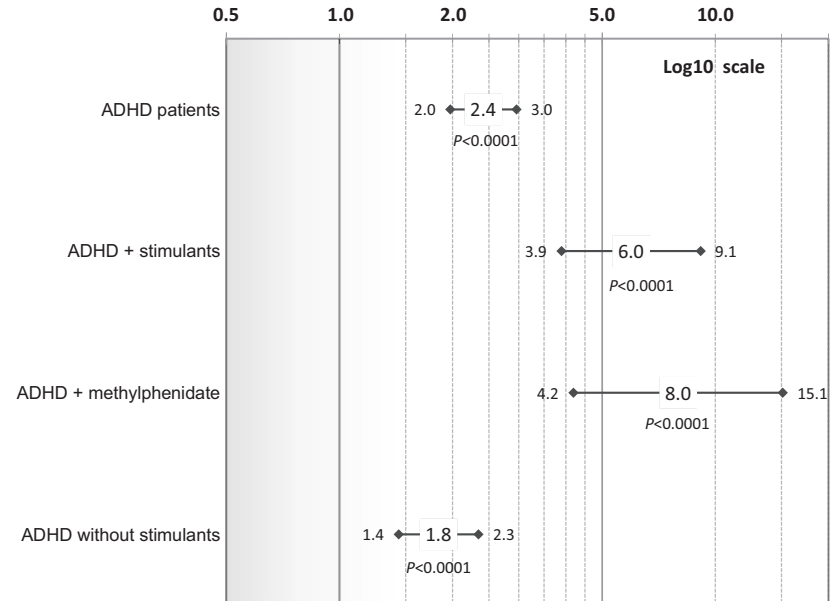

Fig. 1 Risk of basal ganglia and cerebellum diseases (diagnosis age of 21-66 years) in ADHD patients vs. non-ADHD subjects overall and for: ADHD plus (+) stimulants, ADHD plus $(+)$ methylphenidate, and ADHD without known stimulants. Adjusted hazard ratios and $95 \%$ confidence intervals (controlling for sex, age, race/ethnicity, psychotic conditions, tobacco use, and an interaction of psychotic conditions and $\mathrm{ADHD}$ ) are shown on a log 10 scale. Corrected $P$ values are presented (significance level $\leq 0.005$ )

disease, is associated with a history of ADHD, after controlling for independent risks conferred by the presence of psychotic comorbidities (as proxy for antipsychotics) or tobacco use. Although long-standing tobacco prescriptions in Utah have resulted in smoking rates half that of the national average [35], our findings suggest that tobacco use did not protect against Parkinson's risk in those diagnosed with ADHD relative to nonADHD persons. It should be noted that, while some reports suggest tobacco use protects against BG\&C diseases in the general population $[30,31]$, there are other studies that challenge this conclusion based on incorrect interpretation of epidemiological data [32]. Additional studies to elucidate underlying mechanisms are warranted.

While our observational study was not designed to identify biological mechanisms underlying the observed correlation

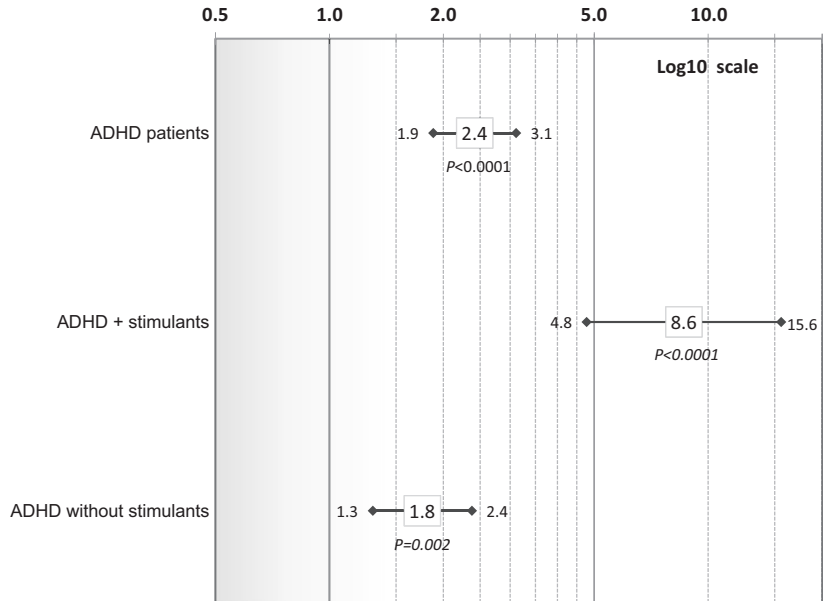

Fig. 2 Risk of earlier-onset basal ganglia and cerebellum diseases diseases (diagnosis age of 21-49 years) in ADHD patients vs. nonADHD subjects overall and for: ADHD plus $(+)$ stimulants and ADHD without known stimulants. Adjusted hazard ratios and $95 \%$ confidence intervals (controlling for sex, age, race/ethnicity, psychotic conditions, tobacco use, and an interaction of psychotic conditions and ADHD) are shown on a log 10 scale. Corrected $P$ values are presented (significance level $\leq 0.005$ )

between $A D H D$ and increased risk of $B G \& C$ diseases including Parkinson's disease, it is noteworthy that these conditions have been associated with dysfunctional central nervous system dopaminergic projections [36,37], suggesting common pathway linkages. It is also of note that the substantia nigra (i.e., the cell body region of nigrostriatal dopaminergic neurons) has been reported to be structurally abnormal in children diagnosed with attention-deficit hyperactivity disorder [38, 39]. It is interesting to speculate that hyperdopaminergic activity, as reported preclinically in at least one model of ADHD (i.e., dopamine transporter knockdown mice [40]), may precede the development of Parkinson's disease, as preclinical electrophysiologic recordings in at least one mouse model of pre-manifest Parkinson's disease (i.e., a-synuclein mutant mice) indicate that hyperdopaminergic activity may serve as a biomarker of dopaminergic neurons that 


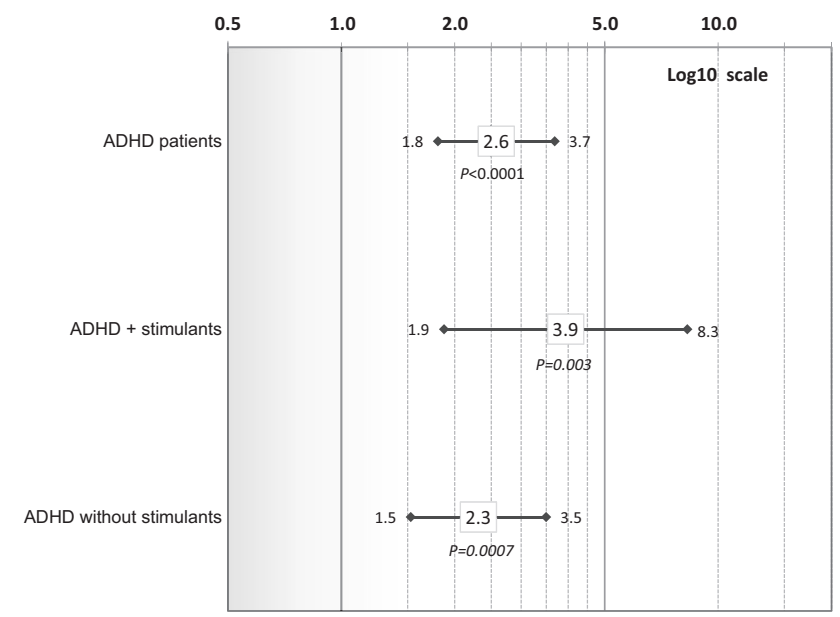

Fig. 3 Risk of Parkinson's disease in ADHD patients vs. non-ADHD subjects overall and for: ADHD plus $(+)$ stimulants and ADHD without known stimulants. Adjusted hazard ratios and $95 \%$ confidence intervals (controlling for sex, age, race/ethnicity, psychotic conditions, tobacco use, and an interaction of psychotic conditions and ADHD) are shown on a log 10 scale. Corrected $P$ values are presented (significance level $\leq 0.005$ )

are vulnerable to damage prior to the onset of degeneration [41]. (Interestingly, increased serotonergic innervation might also contribute to clinical differences in at least one Parkinson's disease phenotype (i.e., that involving leucine-rich repeat kinase 2 [42].) Importantly, no common genetic associations have been found to date between ADHD and PD [43], although future assessment of this possibility is needed.

It is noteworthy that while our investigation includes covariate data of subjects to account for potential confounders such as tobacco use and psychotic disorders, there are other potential confounders such as head trauma/brain injuries and environmental toxins that may contribute to the development of both Parkinson's disease and ADHD [44, 45]. Again, additional investigation as to the contribution of these factors to our present findings is warranted.

We investigated whether restricting individuals with an ADHD history to those known to be prescribed methylphenidate and mixed-AMPH salts altered our observations. Regardless of model, a consistent pattern of significantly increased risk of BG\&C diseases in this subset of stimulant-treated patients was detected. Possible explanations include: (1) treatment with psychostimulants may enhance the mechanism(s) responsible for the linkage between earlier-onset ADHD and BG\&C diseases expression, (2) a history of ADHD with psychostimulant use may accelerate the temporal related degeneration of the relevant neuronal pathways primarily in those patients who eventually, with age, will manifest this disorder regardless of an ADHD history, or (3) psychostimulant treatment is a marker for a more severe ADHD phenotype, which in turn increases the risk of earlier BG\&C diseases expression. Due to a general lack of evidence in either animals $[46,47]$ or humans [48] that methylphenidate per se damages nigrostriatal dopaminergic neurons, the latter explanation may be the most plausible (however see also ref. [49]).

To avoid a potential source of confounding and to minimize selection bias that may arise between the ADHD patient and nonADHD subject cohorts, subjects selected for study did not have an ICD-9 diagnosis history of alcohol abuse or illicit drug abuse. This raises the possibility that casual use or chronic abuse of these substances, in general, may be a common factor that predisposes individuals to Parkinson's. Additional studies are required to examine this possibility. Further investigation examining use and abuse of other substances is also warranted.

Strengths of our investigation include access to populationbased non-ADHD individuals, comprehensive statewide records, and lengthy follow-up. Accordingly, we generally know that nonADHD subjects were living in Utah over the same exposure period as our ADHD cohort members. Our study had access to both statewide inpatient and comprehensive outpatient records from the largest healthcare providers of patient services in Utah. It is important to note that ADHD has been a recognized disorder since the 1970s and our comprehensive EMR came online in 1996. It is likely the actual age of ADHD onset for many patients in this cohort occurred earlier than the index diagnosis age in our ADHD cohort (median 26 years). Limitations include bias from potential misclassification of non-ADHD subjects who did not have an ADHD history in the EMR, but who may have been diagnosed outside of Utah or have undiagnosed ADHD, and patients who may have been misdiagnosed as having ADHD. Administrative claims data may be limited in their ability to identify BG\&C diseases including Parkinson's, and there is no definitive diagnostic test for BG\&C diseases such as Parkinson's disease in living patients. Studies evaluating the accuracy of ICD-9 billing codes to correctly identify clinically recognized Parkinson's disease are mixed as previously discussed [13]. To identify incident outcomes, we used an inclusive, multiple-code approach [50] (hence, BG\&C diseases) to increase sensitivity as studies suggest as many as $3-10 \%$ of all Parkinson's patients experience symptoms before age 40 years $[25,26]$. We acknowledge misclassification of subjects may have occurred. However, when restricted to Parkinson's disease only (ICD-9 332.0), patterns of similar increased risk in ADHD patients were observed. Although the study was restricted to a limited number of planned comparisons, our findings remained statistically significant even after a Bonferroni correction for multiple tests.

Assuming all subjects had 21 years of follow-up, the crude incidence of onset of BG\&C diseases such as Parkinson's disease in younger individuals (aged 21-49 years) in our non-ADHD subjects was 1.2 per 100,000 person years (1.7 per 100,000 person years for combined ADHD patients and non-ADHD subjects), calculated from Table 3. This is consistent with reported incidence of Parkinson's disease onset at ages 30-49 years of $1.4-3.0$ per 100,000 person years [51]. The prevalence of ADHD in the adult population of this study was $1.7 \%$ (31,769 patients of 1.92 million individuals), considerably less than CDC-estimated ADHD prevalence in children and adolescents of $11 \%$ in the United States and $8.3 \%$ in Utah based on 2011 data [15]. This is likely due to the fact that in the past 40 years since ADHD was defined, awareness of a clinical diagnosis has increased and methods of identifying ADHD patients have become more efficient and precise.

In summary, we report that an ADHD diagnosis may increase the risk for future expression of BG\&C diseases, after accounting for potential confounders of race/ethnicity, psychotic conditions, or tobacco use histories. Risk of young onset BG\&C diseases before age 50 years appeared to be enhanced in patients with an ADHD history, particularly in those known to be prescribed stimulants (>8-fold; see Fig. 2). Because ADHD was not widely acknowledged as a psychiatric diagnosis until DSM II in 1968 and is usually identified in children, our ability to assess a linkage between ADHD and BG\&C diseases (typically diagnosed in older adults) was limited to those who developed onset of these neurological conditions before age 66 years and our calculations likely underestimate the total impact of $A D H D$ on the risk for developing $B G \& C$ diseases, especially in younger populations. Consequently, we can report at this time that ADHD, particularly when coupled with psychostimulant treatment, may dramatically increase the risk for expression of earlier-onset BG\&C diseases. These findings also suggest that it is important to assess the possibility of broader significance in follow-up studies by determining if a similar connection exists between ADHD and $B G \& C$ diseases diagnosed in older individuals. Preclinical investigations to identify potential underlying pathology and causative factors are warranted $[4,15,21]$. 


\section{ACKNOWLEDGEMENTS}

We gratefully acknowledge the thoughtful insights and suggestions of Dr. Kathleen Ries Merikangas, Ph.D., and Senior Investigator and Chief, Genetic Epidemiology Research Branch with the National Institute of Mental Health Intramural Research Program in the preparation of this manuscript. We thank the Pedigree and Population Resource, Huntsman Cancer Institute, at the University of Utah (funded in part by the Huntsman Cancer Foundation) for its role in the ongoing collection, maintenance and support of the UPDB. We acknowledge the University of Utah's Information Technology Services and Biomedical Informatics Core for establishing the Master Subject Index between the UPDB, University of Utah Healthcare, and Intermountain Healthcare; without their institutional support, this study would not be possible. The support for this study was provided by National Institute on Drug Abuse grants DA031833 (to G.R.H.) and DA039145 (to A. E.F.). Partial support for the UPDB was provided by: National Cancer Institute P30 CA2014; the University of Utah's Program in Personalized Health and Center for Clinical and Translational Science (funded by NIH Clinical and Translational Science Awards); and a National Center for Research Resources grant, "Sharing Statewide Health Data for Genetic Research" (RR021746 to G. Mineau) with additional support from the Utah State Department of Health.

\section{ADDITIONAL INFORMATION}

Supplementary Information accompanies this paper at (https://doi.org/10.1038/ s41386-018-0207-5).

Competing interest: The authors declare no competing interest.

Publisher's note: Springer Nature remains neutral with regard to jurisdictional claims in published maps and institutional affiliations.

\section{REFERENCES}

1. Bonvicini C, Faraone SV, Scassellati C. Attention-deficit hyperactivity disorder in adults: a systematic review and meta-analysis of genetic, pharmacogenetic and biochemical studies. Mol Psychiatry. 2016;21:872-84.

2. Simon V, Czobor P, Balint S, Meszaros A, Bitter I. Prevalence and correlates of adult attention-deficit hyperactivity disorder: meta-analysis. $\mathrm{Br} \mathrm{J}$ Psychiatry. 2009;194:204-11.

3. Asherson P, Buitelaar J, Faraone SV, Rohde LA. Adult attention-deficit hyperactivity disorder: key conceptual issues. Lancet Psychiatry. 2016;3:568-78.

4. Visser SN, Danielson ML, Wolraich ML, Fox MH, Grosse SD, Valle LA, et al. Vital Signs: National and State-Specific Patterns of Attention Deficit/Hyperactivity Disorder Treatment Among Insured Children Aged 2-5 Years-United States, 2008-2014. MMWR Morb Mortal Wkly Rep. 2016;65:443-50.

5. Halpin LE, Collins SA, Yamamoto BK. Neurotoxicity of methamphetamine and 3,4methylenedioxymethamphetamine. Life Sci. 2014;97:37-44.

6. McCann UD, Kuwabara H, Kumar A, Palermo M, Abbey R, Brasic J, et al. Persistent cognitive and dopamine transporter deficits in abstinent methamphetamine users. Synapse. 2008;62:91-100.

7. McCann UD, Wong DF, Yokoi F, Villemagne V, Dannals RF, Ricaurte GA. Reduced striatal dopamine transporter density in abstinent methamphetamine and methcathinone users: evidence from positron emission tomography studies with $\left[{ }^{11} \mathrm{C}\right] \mathrm{WIN}-35,428$. J Neurosci. 1998;18:8417-22.

8. Sekine $Y$, lyo $M$, Ouchi $Y$, Matsunaga $T$, Tsukada $H$, Okada $H$, et al. Methamphetamine-related psychiatric symptoms and reduced brain dopamine transporters studied with PET. Am J Psychiatry. 2001;158:1206-14.

9. Wilson JM, Kalasinsky KS, Levey Al, Bergeron C, Reiber G, Anthony RM, et al. Striatal dopamine nerve terminal markers in human, chronic methamphetamine users. Nat Med. 1996;2:699-703.

10. Christine CW, Garwood ER, Schrock LE, Austin DE, McCulloch CE. Parkinsonism in patients with a history of amphetamine exposure. Mov Disord. 2010;25:228-31.

11. Garwood ER, Bekele W, McCulloch CE, Christine CW. Amphetamine exposure is elevated in Parkinson's disease. Neurotoxicology. 2006;27:1003-6.

12. Moratalla R, Khairnar A, Simola N, Granado N, Garcia-Montes JR, Porceddu PF, et al. Amphetamine-related drugs neurotoxicity in humans and in experimental animals: main mechanisms. Prog Neurobiol. 2017;155:149-70.

13. Curtin K, Fleckenstein AE, Robison RJ, Crookston MJ, Smith KR, Hanson GR. Methamphetamine/amphetamine abuse and risk of Parkinson's disease in Utah: a population-based assessment. Drug Alcohol Depend. 2015;146:30-8.

14. Meszaros A, Czobor P, Balint S, Komlosi S, Simon V, Bitter I. Pharmacotherapy of adult attention deficit hyperactivity disorder (ADHD): a meta-analysis. Int J Neuropsychopharmacol. 2009;12:1137-47.

15. Visser SN, Danielson ML, Bitsko RH, Holbrook JR, Kogan MD, Ghandour RM, et al. Trends in the parent-report of health care provider-diagnosed and medicated attention-deficit/hyperactivity disorder: United States, 2003-2011. J Am Acad Child Adolesc Psychiatry. 2014;53:34-46 e32.

16. Post RE, Kurlansik SL. Diagnosis and management of adult attention-deficit/ hyperactivity disorder. Am Fam Physician. 2012;85:890-6.

17. Benson K, Flory K, Humphreys KL, Lee SS. Misuse of stimulant medication among college students: a comprehensive review and meta-analysis. Clin Child Fam Psychol Rev. 2015;18:50-76.

18. Willis AW, Schootman M, Kung N, Racette BA. Epidemiology and neuropsychiatric manifestations of Young Onset Parkinson's Disease in the United States. Park Relat Disord. 2013;19:202-6.

19. Tse W, Cersosimo MG, Gracies JM, Morgello S, Olanow CW, Koller W. Movement disorders and AIDS: a review. Park Relat Disord. 2004;10:323-34.

20. Callaghan RC, Cunningham JK, Sykes J, Kish SJ. Increased risk of Parkinson's disease in individuals hospitalized with conditions related to the use of methamphetamine or other amphetamine-type drugs. Drug Alcohol Depend. 2012;120:35-40.

21. Brecht ML, Huang D, Evans $E$, Hser YI. Polydrug use and implications for longitudinal research: ten-year trajectories for heroin, cocaine, and methamphetamine users. Drug Alcohol Depend. 2008;96:193-201.

22. Bettiol SS, Rose TC, Hughes CJ, Smith LA. Alcohol consumption and Parkinson's disease risk: a review of recent findings. J Park Dis. 2015;5:425-42.

23. Hernan MA, Chen $\mathrm{H}$, Schwarzschild MA, Ascherio A. Alcohol consumption and the incidence of Parkinson's disease. Ann Neurol. 2003;54:170-5.

24. Liu R, Guo X, Park Y, Wang J, Huang X, Hollenbeck A, et al. Alcohol consumption, types of alcohol, and Parkinson's disease. PLoS ONE. 2013;8:e66452.

25. Schrag A, Schott JM. Epidemiological, clinical, and genetic characteristics of earlyonset parkinsonism. Lancet Neurol. 2006;5:355-63.

26. Uc EY, Rodnitzky RL. Juvenile parkinsonism. Semin Pediatr Neurol. 2003;10:62-7.

27. Kleinbaum DG, Klein M. Competing risks survival analysis. New York, NY: Springer; 2005. p. 391-461.

28. Dalsgaard S, Ostergaard SD, Leckman JF, Mortensen PB, Pedersen MG. Mortality in children, adolescents, and adults with attention deficit hyperactivity disorder: a nationwide cohort study. Lancet. 2015;385:2190-6.

29. Howes OD, Murray RM. Schizophrenia: an integrated sociodevelopmentalcognitive model. Lancet. 2014;383:1677-87.

30. Hernan MA, Takkouche B, Caamano-Isorna F, Gestal-Otero JJ. A meta-analysis of coffee drinking, cigarette smoking, and the risk of Parkinson's disease. Ann Neurol. 2002;52:276-84.

31. Wirdefeldt K, Adami HO, Cole P, Trichopoulos D, Mandel J. Epidemiology and etiology of Parkinson's disease: a review of the evidence. Eur J Epidemiol. 2011;26:S1-58.

32. Ritz B, Lee PC, Lassen CF, Arah OA. Parkinson disease and smoking revisited: ease of quitting is an early sign of the disease. Neurology. 2014:83:1396-402.

33. Shin HW, Chung SJ. Drug-induced parkinsonism. J Clin Neurol. 2012;8:15-21.

34. Sandoval V, Riddle EL, Hanson GR, Fleckenstein AE. Methylphenidate alters vesicular monoamine transport and prevents methamphetamine-induced dopaminergic deficits. J Pharmacol Exp Ther. 2003;304:1181-7.

35. Merrill RM, Hilton SC, Daniels M. Impact of the LDS church's health doctrine on deaths from diseases and conditions associated with cigarette smoking. Ann Epidemiol. 2003;13:704-11.

36. Brichta L, Greengard P. Molecular determinants of selective dopaminergic vulnerability in Parkinson's disease: an update. Front Neuroanat. 2014;8:152.

37. Swanson JM, Kinsbourne M, Nigg J, Lanphear B, Stefanatos GA, Volkow N, et al. Etiologic subtypes of attention-deficit/hyperactivity disorder: brain imaging, molecular genetic and environmental factors and the dopamine hypothesis. Neuropsychol Rev. 2007;17:39-59.

38. Krauel K, Feldhaus HC, Simon A, Rehe C, Glaser M, Flechtner HH, et al. Increased echogenicity of the substantia nigra in children and adolescents with attentiondeficit/hyperactivity disorder. Biol Psychiatry. 2010;68:352-8.

39. Romanos M, Weise $D$, Schliesser M, Schecklmann M, Loffler J, Warnke A, et al. Structural abnormality of the substantia nigra in children with attention-deficit hyperactivity disorder. J Psychiatry Neurosci. 2010;35:55-58.

40. Zhuang X, Oosting RS, Jones SR, Gainetdinov RR, Miller GW, Caron MG, et al. Hyperactivity and impaired response habituation in hyperdopaminergic mice. Proc Natl Acad Sci USA. 2001;98:1982-7.

41. Subramaniam M, Althof D, Gispert S, Schwenk J, Auburger G, Kulik A, et al. Mutant alpha-synuclein enhances firing frequencies in dopamine substantia nigra neurons by oxidative impairment of A-type potassium channels. J Neurosci. 2014;34:13586-99.

42. Wile DJ, Agarwal PA, Schulzer M, Mak E, Dinelle K, Shahinfard E, et al. Serotonin and dopamine transporter PET changes in the premotor phase of LRRK2 parkinsonism: cross-sectional studies. Lancet Neurol. 2017;16:351-9.

43. Geissler JM, International Parkinson Disease Genomics Consortium, Romanos M, Gerlach M, Berg D, Schulte C. No genetic association between attention-deficit/ 
hyperactivity disorder (ADHD) and Parkinson's disease in nine ADHD candidate SNPs. Atten Defic Hyperact Disord. 2017;9:121-7.

44. Bjorklund G, Stejskal V, Urbina MA, Dadar M, Chirumbolo S, Mutter J. Metals and Parkinson's disease: mechanisms and biochemical processes. Curr Med Chem. 2018;25:2198-2214 (Epub ahead of print).

45. Camacho-Soto A, Warden MN, Searles Nielsen S, Salter A, Brody DL, Prather H et al. Traumatic brain injury in the prodromal period of Parkinson's disease: a large epidemiological study using medicare data. Ann Neurol. 2017;82: 744-54.

46. Volkow ND. Long-term safety of stimulant use for ADHD: findings from nonhuman primates. Neuropsychopharmacology. 2012;37:2551-52.

47. Yuan J, McCann U, Ricaurte G. Methylphenidate and brain dopamine neurotoxicity. Brain Res. 1997;767:172-5.
48. Wang GJ, Volkow ND, Wigal T, Kollins SH, Newcorn JH, Telang F, et al. Longterm stimulant treatment affects brain dopamine transporter level in patients with attention deficit hyperactive disorder. PLoS ONE. 2013;8: e63023.

49. Sadasivan S, Pond BB, Pani AK, Qu C, Jiao Y, Smeyne RJ. Methylphenidate exposure induces dopamine neuron loss and activation of microglia in the basal ganglia of mice. PLoS ONE. 2012;7:e33693.

50. Noyes K, Liu H, Holloway R, Dick AW. Accuracy of Medicare claims data in identifying Parkinsonism cases: comparison with the Medicare current beneficiary survey. Mov Disord. 2007;22:509-14.

51. Van Den Eeden SK, Tanner CM, Bernstein AL, Fross RD, Leimpeter A, Bloch DA et al. Incidence of Parkinson's disease: variation by age, gender, and race/ethnicity. Am J Epidemiol. 2003;157:1015-22. 